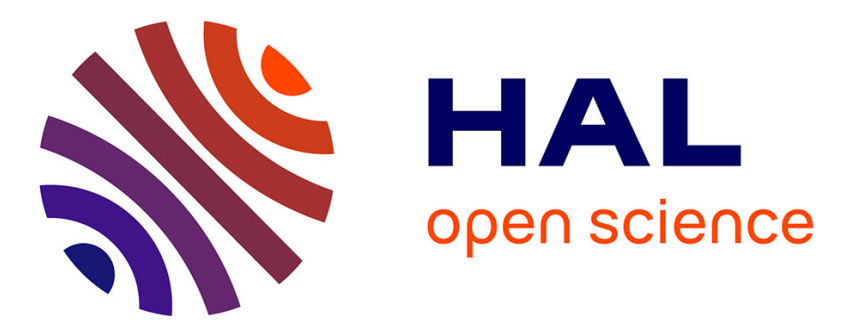

\title{
Alterations in rat adipose tissue transcriptome and proteome in response to prolonged fasting
}

Marianne Ibrahim, Daniel Ayoub, Thierry Wasselin, Alain van Dorsselaer, Yvon Le Maho, Thierry Raclot, Fabrice Bertile

\section{- To cite this version:}

Marianne Ibrahim, Daniel Ayoub, Thierry Wasselin, Alain van Dorsselaer, Yvon Le Maho, et al.. Alterations in rat adipose tissue transcriptome and proteome in response to prolonged fasting. Biological Chemistry, 2020, 10.1515/hsz-2019-0184 . hal-02333569

\section{HAL Id: hal-02333569 \\ https://hal.science/hal-02333569}

Submitted on 9 Oct 2020

HAL is a multi-disciplinary open access archive for the deposit and dissemination of scientific research documents, whether they are published or not. The documents may come from teaching and research institutions in France or abroad, or from public or private research centers.
L'archive ouverte pluridisciplinaire HAL, est destinée au dépôt et à la diffusion de documents scientifiques de niveau recherche, publiés ou non, émanant des établissements d'enseignement et de recherche français ou étrangers, des laboratoires publics ou privés. 
Alterations in rat adipose tissue transcriptome and proteome in response to prolonged fasting

Running title: Molecular responses of adipose tissue to fasting

Marianne Ibrahim ${ }^{1,2}$, Daniel Ayoub ${ }^{1,2, a}$, Thierry Wasselin ${ }^{1,2, b}$, Alain Van Dorsselaer ${ }^{1,2}$, Yvon Le Maho $^{1,3}$, Thierry Raclot ${ }^{1,3}$, Fabrice Bertile*1,2

${ }^{1}$ Université de Strasbourg, CNRS, IPHC UMR 7178, F-67000 Strasbourg, France.

${ }^{2}$ Laboratoire de Spectrométrie de Masse Bio-Organique, 25 rue Becquerel, F-67087 Strasbourg, France.

${ }^{3}$ Département Ecologie, Physiologie, Ethologie, 23 rue Becquerel, F-67087 Strasbourg, France.

* Correspondence to:

Fabrice Bertile, Université de Strasbourg, CNRS, IPHC UMR 7178, F-67087 Strasbourg, France, e-mail : fbertile@unistra.fr, phone: +33 368852681 , fax: +33 368852781.

a Present address: Glenmark Pharmaceuticals S.A., La Chaux-de-Fonds, Switzerland

${ }^{b}$ Present address: Department of Clinical Chemistry, University Medical Center, Göttingen, Germany 


\section{Abstract}

Various pathophysiological situations of negative energy balance involve the intense depletion of body energy reserves. White adipose tissue is a central place to energy storage and a major endocrine organ. As a model of choice to better understand how the white adipose tissue dynamically responds to changes in substrate availability, we used the prolonged fasting paradigm, which is characterized by successive periods of stimulated (phase 2) and then reduced (phase 3) lipid mobilization/utilization. Using omics analyses, we report a regulatory transcriptional program in rat epididymal adipose tissue favoring lipolysis during phase 2 and repressing it during phase 3 . Changes in gene expression levels of lipases, lipid dropletassociated factors, and the proteins involved in cAMP-dependent and CAMP-independent regulation of lipolysis were highlighted. The mRNA and circulating levels of adipose-secreted factors were consistent with the repression of insulin signalling during prolonged fasting. Other molecular responses are discussed, including the regulation of leptin and adiponectin levels, the specific changes reflecting an increased fibrinolysis and a possible protein catabolism-related energy saving mechanism in late fasting. Finally, few differences between internal and subcutaneous adipose tissues are also reported. These data provide a comprehensive molecular basis of adipose tissue responses when facing a major energetic challenge.

\section{Keywords}

Fasting, omics, rat, lipolysis, adipokines, hemostasis 


\section{Introduction}

Fat tissue is a major regulator of whole-body energy homeostasis (Luo and Liu, 2016). As the main energy reservoir, adipose tissue controls the balance between storage and release of lipids in response to altered energy intake or expenditure to meet bioenergetics needs. During periods of excess energy intake, triglycerides are efficiently deposited into cytosolic droplets, which may result in obesity and related diseases. Conversely, triglycerides are hydrolysed during times of increased energy demand to release fatty acids to the circulation. Adipose tissue is also an important endocrine organ (Bluher and Mantzoros, 2015; Fasshauer and Bluher, 2015), producing a number of adipokines/cytokines that regulate overall metabolism, insulin sensitivity, endothelial function, hemostasis, and the immune system. In relation to the catabolic pathophysiological situations where the level of fat store depletion may greatly differ, a better understanding of the adipose molecular response to nutritional and metabolic transitions would be helpful to gain insights into the regulation of energy substrate availability. Up to now, the molecular mechanisms of adipose tissue have been investigated mainly during feeding and short/medium fasting (Bertile et al., 2003; Bertile and Raclot, 2004b; Ding et al., 2016; Duncan et al., 2007; Duszka et al., 2013; Lafontan and Langin, 2009; Li et al., 2006; Palou et al., 2010; Schupp et al., 2013; Sethi and Vidal-Puig, 2007; Viscarra and Ortiz, 2013; L. Yang et al., 2011), i.e. in situations where energy metabolism relies mostly on lipid availability. During prolonged fasting, however, three sequential metabolic phases have been described in humans (Owen et al., 1998), non-hibernating mammals (Bertile and Raclot, 2008), and birds (Groscolas and Robin, 2001). The required energy is at first derived from glucose and lipids during a short period of adaptation (phase 1), while the net catabolism of body proteins is decreased. As the fast duration is increased, energy needs are met mostly from the use of lipid fuels during phase 2, while proteins are efficiently spared. Finally, before exhaustion of fat 
stores, proteins are increasingly used for energy generation during phase 3 . Protein catabolism is one of the determinants of the safety limit of fasting (Bertile et al., 2016). Characterized by successive periods of stimulated and then reduced lipid mobilization/utilization, the prolonged fasting paradigm is therefore a good model to gain insights into the molecular mechanisms underlying the nutritional regulation of adipose lipid metabolism. It is also well known that nutritional transitions can alter the gene expression and/or plasma levels of adipose-secreted factors (Bertile and Raclot, 2004a, 2006). In the context of the metabolic phases of prolonged fasting, very few studies have analysed the expression levels of key adipose metabolic factors providing fuel substrates, neither those of secreted proteins acting as bioactive molecules possibly signalling nutritional and/or metabolic changes (Bertile and Raclot, 2004a, 2006, 2011; Li et al., 2006). Therefore, further research is needed to better assess the molecular regulations that link white adipose tissue to fuel partitioning and the level of fat depletion during long term fasting.

High-throughput technologies, the so-called omics, nowadays have taken front stage in life science and medical research to investigate global cellular and molecular responses and better understand diseases or pathophysiological situations (Bertile and Raclot, 2008; Manzoni et al., 2016). The response to short term fasting has already been investigated in different tissues, including in white adipose tissue at the transcriptomic (Duszka et al., 2013; Faulconnier et al., 2011; Ji et al., 2012; Li et al., 2006; Schupp et al., 2013) and proteomic (L. Yang et al., 2011) levels. Adipose transcriptome response to prolonged fasting has been described in elephant seal pups during prolonged fasting, but their metabolic state, i.e. whether they were in phase 2 or phase 3, was not assessed (Martinez et al., 2018). Yet, omics data according to the metabolic phases of prolonged fasting have only been reported in the rat liver (Wasselin et al., 2014) and rat and penguin plasma (Bertile et al., 2009; Bertile et al., 2016). To better 
understand how the white adipose tissue responds to changes in energy substrate availability and gradual levels of fat store depletion, the present study analysed how the adipose transcriptome and proteome are affected during prolonged fasting. Specific aims were to determine whether i) specific regulation of adipose lipolytic-related factors could be responsible for low fat mobilization in the late stage of fasting and ii) regulation of adipose secreted factors could help draw hypotheses in relation to the increased drive for refeeding in late fasting and/or to possible autocrine/paracrine/endocrine effects contributing to the adaptation to food deprivation. As adipose tissue exhibit regional differences in response to fasting (Bertile et al., 2003; Bertile and Raclot, 2004a, 2006, 2011; Palou et al., 2010), we investigated the response to prolonged food deprivation of visceral and subcutaneous (SC) adipose tissues. Finally, because several adipose-derived factors are involved in the control of hemostasis, we also explored their levels in the plasma of fed and fasted rats. 


\section{Results}

\section{Animal metabolic status}

Animal metabolic state was determined using variations in body mass loss and urea circulating levels (Table 1; $\mathrm{N}=8$ /group). Initial body mass was similar in the three experimental groups. Rats in phase 3 of fasting significantly lost more body weight (33\%) than rats in phase 2 (23\%) compared to fed animals ( $p$-value $=0.000015)$. The utilization of mainly lipid fuels during phase 2 of fasting and mobilization of body proteins in phase 3 was reflected in the increase of daily rate of body mass loss $(\mathrm{dm} / \mathrm{m}$.dt; $\mathrm{p}$-value $=0.028)$ and plasma urea levels $(\mathrm{p}$-value $=$ 0.0002 ) in phase 3 relative to phase 2 of fasting. In addition, mobilization of lipid reserves during fasting triggered a significant drop in the mass of epididymal adipose tissue (EPI), which was 1.8 times more pronounced in phase 3 than phase 2 of fasting ( $74 \%$ vs. $42 \%$; $p$-value $=$ 0.00045). The plasma levels of key metabolites (glucose and fatty acids) and hormones/cytokines (NPY, insulin, leptin, TNF $\alpha$, and adiponectin) connected to our omics data were measured here (see below). It is of note that the levels of other plasma metabolites and hormones have been reported elsewhere (Bertile and Raclot, 2004a; Bertile et al., 2009; Bertile and Raclot, 2011; Wasselin et al., 2014) in similar fed and fasted rats.

\section{Overall EPI adipose tissue transcriptome changes in response to prolonged fasting}

Based on four biological replicates per group, and after elimination of reference genes, microarray analysis considered signal intensities from 26788 probes (see Table S1). The signals from a total of 11674 probes were differentially expressed between groups (ANOVA p-value below 0.05 , which corresponded to a false discovery rate [FDR] of 0.078 ). Thus, a very pronounced change of the overall EPI transcriptome was due to fasting. The overlap of probes 
with changing signals $(P<0.05)$ depending on the group considered revealed that the vast majority of them (82\%) were differential in phase $3 \mathrm{vs.} \mathrm{the} \mathrm{fed} \mathrm{state,} \mathrm{with} \mathrm{an} \mathrm{approximately}$ equivalent number of probe signals being up- and downregulated. Half of the 11674 probes exhibited a differential signal in phase $2 v s$. the fed state, of which $42 \%$ were upregulated and $58 \%$ were downregulated. In addition, half of the 11674 probes also exhibited a differential signal in phase 3 vs. phase 2 of fasting, of which $48 \%$ were upregulated and $52 \%$ were downregulated. Because the number of differentially-regulated transcripts was high when considering an ANOVA p-value threshold of 0.05 , we checked the data by using also a $p$-value threshold of 0.0001 . The number of differential signals then dropped to 2870 probes $(P<0.0001 ;$ corresponding to a $F D R=0.008 ;$ Figure $1 A)$. Of them, $92 \%$ were differential in phase 3 and $31 \%$ in phase 2 vs. the fed state (with $\sim 2$ times more down- than up-regulations). Moreover, 34\% were differential between fasting stages, with an approximately equivalent number of down- and up-regulated genes. Altogether, these results illustrate that changes were much more pronounced in phase 3 than in phase 2.

Hierarchical clustering analyses of genes and arrays were carried out for the set of differential probe signals when considering statistical p-values below 0.0001 (Figure 1B). Clustering data indicate low variability between biological replicates. Genes were hence successfully distinguished between groups, with mRNA expression profiles in fed animals being well distinguished from those of fasting stages.

\section{Pathway analysis of EPI adipose tissue transcriptome responses to fasting}

To further investigate the main transcriptomics effects of prolonged fasting, functional annotation analysis focused on EPI transcripts that were the most significantly changed (i.e. those with p-values below 0.0001 ) across groups. A total number of 63,118 , and 4 relevant 
GO terms were found to be significantly enriched when comparing phase 2 to fed, phase 3 to fed, and phase 3 to phase 2 animals, respectively (Table S2). These GO terms were distributed into several pathways and functions, notably including metabolism, tissue remodelling, the endoplasmic reticulum function, and the response to oxidative stress (Figure 1C-E). Apart from several broad functions and pathways like fuel metabolism that were significantly affected by both fasting stages, we observed that phase 3 triggered specific changes, notably related to the response to oxidative stress, angiogenesis, PI3K signalling, and protein metabolism.

A closer look at the most significant changes (Figure 2A) revealed that, compared to the fed state, prolonged fasting significantly decreased the abundance of five transcripts coding antioxidant enzymes (up to 3-fold in phase 3 of fasting7), while the abundance of only Gpx3 and Sqstm1 was increased (2-fold in phase 3). Nineteen EPI transcripts involved in carbohydrate metabolism were downregulated by prolonged fasting compared to the fed state, in both phase 2 and phase 3 (1.5-14-fold) while only the abundance of Gulo and B3galt1 was increased (5-18-fold in phase 2 and phase 3). Regarding lipid metabolism, changes were globally more marked in phase 3 than in phase 2 of fasting vs. the fed state, with downregulation of six lipid synthesis-related transcripts (1.5-5-fold), adiponectin receptor 2 (Adipor2; 1.4-1.7-fold), and leptin (Lep; 17- and 84-fold in phase 2 and phase 3, respectively). On the contrary, EPI transcripts related to fatty acid oxidation were upregulated in fasting vs. fed animals (2-17-fold in phase 2 and phase 3; Cpt1a, Acacb, Acs/4). The abundance of transcripts coding subunits of the different complexes of the respiratory chain were globally decreased by phase 3 of fasting (1.5-3.3-fold). A lot of transcripts related to proteostasis were regulated by fasting compared to the fed state, with those involved in protein synthesis (chaperones and elongation factors) and those involved in different proteolytic systems (calpains, cathepsins, Sqstm1, COP9 signalosome, and Clpp proteases) being either down- or 
upregulated in phase 2 and especially in phase 3 . It is of note that the abundance of E3 ubiquitin-ligase TRIM63 (also termed MuRF1) was 87 times higher in phase 3 than in the fed state. The other EPI transcripts that were significantly regulated by fasting vs. the fed state (more markedly in phase 3 than phase 2) were for example involved in insulin signalling, tricarboxylic acid cycle and lactate metabolism. Finally we also noticed fasting-induced downregulation of G0s2 and Deptor (2-12-fold in phase 2 and especially in phase 3).

To further identify biomarkers specific to phase 3 of fasting, we examined in more detail expression profiles of changed transcripts (Figure S1). Among biomarkers of metabolic phases of fasting, selected on the basis of the magnitude of variation and known functions, phase 3 was notably characterized by the strong and specific upregulation of argininosuccinate synthetase 1 (Ass1) and downregulation of galectin 12 (Lgals12) and the glycerol transporter Aqp7.

\section{Regulation of EPI genes associated with lipid metabolism in response to prolonged fasting}

Beyond the overall EPI adipose tissue transcriptome changes in response to prolonged fasting, we next focused on lipid metabolism. In accordance with fuel partitioning during prolonged fasting, plasma non esterified fatty acids (NEFA) levels were significantly increased in phase 2 vs. the fed state (2.3-fold), while they were restored to initial values during phase 3 (Figure 3A). The relative abundance of EPI transcripts that play a role in regulation of lipolysis is illustrated in Figure 3A, which is based on the KEGG pathway map rno04923. Regarding the main white adipose tissue lipases, ATGL transcript levels were increased (1.5-fold) in phase 2, while both HSL and monoglyceride lipase (MGL) transcript levels were decreased in phase 3 $(\sim 2$-fold) relative to the fed state. In addition, the minor monoglyceride lipases abhd 6 and Abhd12 were upregulated by fasting relative to the fed state, the former in phase 3 (1.5-fold) 
and the latter in phase 2 (2.2-fold). The fatty acid transport proteins FABP4 and FABPpm, the glycerol transport protein (AQP7), as well as lipid droplet-associated proteins (perilipin-1 and -2 , FSP27, Seipin, Caveolin 1 and 2) were also downregulated especially in phase 3 vs. the fed state $(\sim 1.3-10$-fold $)$.

cAMP-dependent protein kinase subunit levels were either unchanged by fasting (PRKACb) or downregulated (PRKACa) in both phase 2 and phase 3 of fasting vs. the fed state (1.5-2-fold). The regulation of adenylate cyclase depended on the type considered, with downregulation of type 3, 5 and 7 in phase 2 and/or phase 3 of fasting vs. the fed state (1.4-2.6-fold) and upregulation of type 2 and 4 (1.4 times) in phase 3 and phase 2 of fasting, respectively. Adenylate cyclase-stimulating $\mathrm{G}$ alpha protein (GNAS) was downregulated by both fasting stages compared to the fed state (1.3-1.8-fold) while adenylate cyclase-inhibiting G alpha protein GNAI1 was downregulated only in phase 3 (2.2-fold) and GNAI2 and GNAI3 remained unchanged. Relative to the fed state, thyrotropin receptor (TSHR) and beta adrenergic receptor 3 (ADRB3) transcript levels were decreased only in phase 3 of prolonged fasting (6.3and 2.6-fold, respectively), while the beta adrenergic receptor 2 isoform was upregulated in both fasting stages vs. the fed state (1.3-fold). In comparison with the fed state, the adipocyte receptors for prostaglandin E2 (PTGER3) and adenosine (ADORA1) were downregulated in phase 2 and phase 3 of fasting (1.6-4.2-fold), while the neuropeptide $Y$ receptor type 1 (NPY1R) was on the contrary upregulated in phase 2 (1.6-fold) and phase 3 of fasting (3.3fold). At the plasma level, NPY concentrations were found to significantly decrease in phase 3 of fasting relative to the fed state (2-fold).

Among proteins that are known to have an inhibitory effect on ATGL and HSL, G0/G1 switch gene 2 (GOS2) was downregulated in phase 2 (1.8-fold) and even more in phase 3 (12-fold) vs. the fed state, protein phosphatase-1 subunits were either downregulated in phase 3 (PP1a; 
1.5-fold) or upregulated by both fasting stages (PP1b; 1.2-1.4-fold), phospholipase C-Related Catalytically Inactive Protein (PRIP) was upregulated in phase 2 and phase 3 of fasting (1.41.8-fold), and AMP-activated kinase (AMPK) subunits alpha-2, beta-2 and gamma-1 were downregulated in phase 2 and/or phase 3 of prolonged fasting (alpha-2, beta-2, and gamma1; 1.3-1.9-fold), whereas the AMPK alpha-1 subunit was increased by both fasting phases vs. the fed state (1.9-2.3-fold) and the beta-1 and gamma-2 subunits remained unaffected. We also considered the factors that are involved in insulin cAMP-dependent suppression of lipolysis. Relative to the fed state, plasma insulin levels were significantly decreased in phase 2 (2.6-fold), and even more in phase 3 (3.8-fold). The level of its receptor was found to increase in response to prolonged fasting in phase2 (2.3-fold) and phase 3 (3.4-fold). The levels of insulin receptor substrates 1 and 3 (IRS1/3) remained unchanged, while that of IRS2 was increased in both fasting stages (2.3-2.5-fold). Compared to the fed state, most of phosphatidyl inositol kinase-3 (PI3K) subunits were downregulated by phase 2 and/or phase 3 of prolonged fasting (1.2-1.6-fold), with only the alpha subunit being slightly upregulated (1.2-fold). Protein kinase B/Akt AKT1 was downregulated only in phase 3 vs. the fed state (1.3fold) while AKT2 was downregulated in both phases of prolonged fasting (1.5-3-fold), and AKT3 was upregulated at the same time (1.6-1.8-fold). Phosphodiesterase 3B (PDE3B) was also transitorily upregulated by fasting in phase 2 vs. the fed state (2.5-fold).

Glucose and fatty acids taken up from the circulation are main substrates for lipogenesis. Circulating glucose levels were gradually decreased by fasting in phase 2 (1.4-fold) and phase 3 (1.6-fold) vs. the fed state (Figure 3B). The relative abundance across rat groups of EPI transcripts that play a role in the regulation of lipogenesis is illustrated in Figure 3B. The CD36 fatty acid transporter, very long chain acyl-CoA synthase (ACS), long-chain fatty acid-CoA ligase isozymes (Acsl3, Acsl4, Acslbg1), perilipin-5 (PLIN5), phosphatidate phosphatase-1 (Lpin1), 
and fructose-bisphosphate aldolase C (ALDOC) were upregulated by fasting vs. the fed state (1.3-13.4-fold). All other lipogenic factors were downregulated in both fasting phases vs. the fed state (FASN, GPD1L, GLUT4, Lipin2, DGAT; 1.4-43-fold), or only in phase 2 (Lipin3) or phase 3 (LPL; 1.2-fold). Compared to the fed state, lipogenesis-related transcription factors CEBP alpha and PPAR gamma were also downregulated by phase 3 of fasting (1.7-2.3-fold), while RXR gamma was transitorily increased in phase 2 (1.9-fold).

\section{Regulation of EPI genes encoding secreted factors in response to prolonged fasting}

The relative abundance across rat groups of EPI transcripts coding adipose-secreted factors is illustrated in Figure 4. Expression levels of most of them remained unchanged during fasting, while leptin, retinol-binding protein 4 (RBP4), chemerin (Rarres2), and insulin-like growth factor 1 (IGF1) were decreased in phase 2 (1.4-17.3-fold) and even more markedly in phase 3 of fasting (3-84-fold). Adiponectin (AdipoQ), resistin, adipsin, apelin, and vascular endothelial growth factor B (VEGFb) were decreased by fasting only in phase 3 (1.9-4.7-fold). For the other changing transcripts of secreted factors, we notably observed an upregulation of apolipoprotein E (ApoE), angiopoietin-related protein 4 (ANGPTL4), visfatin and plasminogen activator inhibitor 1 (PAI) in phase $2(\sim 2$-fold) and phase 3 of prolonged fasting vs. the fed state (1.5-4.9-fold). The circulating levels of leptin and TNF $\alpha$ was in line with their adipose transcript levels. Indeed, leptinemia strongly dropped in phase 2 vs. the fed state (54-fold), and plasma leptin was not detectable in phase 3. Plasma TNF $\alpha$ remained unchanged whatever the metabolic phase considered. Changes in adiponectinemia did not reach significance. The adiponectin receptor 2 (ADIPOR2) exhibited a decreased expression due to food deprivation (1.4-1.7-fold). Finally, the adiponectin receptor 1 (ADIPOR1), tumor necrosis factor receptor TNFR1B, IL6 receptor subunit beta, leptin receptor, and type-1A angiotensin II receptor 
(AGTRA) were upregulated in phase 2 (1.2-1.8-fold) and/or phase 3 of fasting (1.3-2.3-fold) vs. the fed state.

\section{EPI and SC adipose tissue proteome changes in response to prolonged fasting}

Investigation of proteome differences between the EPI and SC white adipose tissues of control fed rats and of the changes in EPI and SC in response to prolonged fasting was performed using quantitative proteomics according to the 2D difference gel electrophoresis (DIGE)/mass spectrometry (MS) approach. A total of about 400 proteins spots were nicely detected on 2D DIGE gels (Figure S2), of which 8 exhibited a differential $(p<0.05)$ intensity in EPI vs. SC of control fed rats (Figure S3), 35 in response to fasting vs. the fed state in EPI (Figure 2B) and 37 in SC (Figure S3). Functional annotation analysis using Gene Ontology annotations and literature examination revealed that differences between EPI and SC in control animals involved proteins known to play a role in carbohydrate metabolism (PDHB; two times less abundant in $\mathrm{SC}, \mathrm{p}$-value $=0.02)$, the response to oxidative stress (CA5B; 2.5 less abundant in SC; $p$-value $=0.02$ ), nucleotide metabolism (PNP; two times less abundant in SC; $p$-value $=$ 0.02), the immune response (TAGLN; 1.6 times more abundant in SC; $p$-value $=0.01$ ), coagulation cascade (FGB and FGG; two times less abundant in SC; $p$-value $=0.02$ and 0.01 , respectively), protein metabolism (HSPB1; 1.6 times less abundant in SC; $p$-value $=0.01$ ), and vitamin D metabolism (GC; two times less abundant in SC; $p$-value $=0.03$ ) (Table S3). In EPI (Figure 2B), the abundance of proteins known to play a role in the response to oxidative stress was significantly decreased during phase 2 (CAT, CA3, and CBR1; two-fold; p-value = 0.003-0.04) and even more in phase 3 (CAT, CA3, CBR1, and GSTA3; 2 -4-fold; $p$-value $=0.001$ 0.04) of fasting compared to the fed state. One antioxidant protein was on the contrary upregulated by prolonged fasting vs. the fed state in EPI (PRDX2; 3-3.5-fold; $p$-value $=0.06$ in 
phase 2 and $p$-value $=0.02$ in phase 3 ). In addition, EPI proteins involved in carbohydrate metabolism (PDHB, GAPDH, ALDOA, PKM, MDH1, and TKT) were downregulated ( $p$-value = $0.003-0.05)$ in phase $2(2.5-5$-fold $)$ and even more in phase $3(2.5-10$-fold $)$ relative to the fed state. EPI proteins involved in protein synthesis and folding were also downregulated by fasting, especially in phase 3 vs. the fed state (TUFM, EF1A1, PDIA3, ERP29, and HSPA8; 2-3fold; $p$-value $=0.02-0.04)$. Among others, several EPI proteins downregulated by fasting are known to be involved in lactate metabolism (LDHB; 2.5 -fold in phase 3 , p-value $=0.047$ ), the mitochondrial respiratory chain (ATP5B, ATP5H; 2-3-fold in phase 2 and phase $3, p$-value $=$ 0.004-0.04), and other metabolic-related pathways (see Table S3). Interestingly, one EPI protein involved in lipid metabolism was upregulated in phase 2 of fasting (APOA1; 1.7 -fold; $p$-value $=0.02$ ). During prolonged fasting vs. the fed state, we also observed the upregulation of an EPI protein that may act as an adipocyte differentiation factor (TAGLN; 5-fold in phase 2 and phase $3 ;$-value $=0.01-0.003)$.

In SC (Figure S3), prolonged fasting triggered the downregulation of two proteins relative to values in the fed state (CA3 and PRDX1; 3-10-fold in phase 2 and phase $3 ; p$-value $=0.01-0.04$ ) and the transient upregulation of one protein (GSTM2; 3 -fold in phase 2 ; p-value $=0.03$ ) involved in the response to oxidative stress. The abundance of SC proteins involved in carbohydrate metabolism was reduced in phase 3 vs. the fed state (TPI1, AKR1B1, ENO1, G6PDX, and MDH1; 1.7-14-fold; $p$-value $=0.02-0.04)$. SC proteins involved in protein synthesis and folding were also downregulated in response to prolonged fasting (P4HB, HSPA5, HSP90B1, PDIA3, PDIA6, and PRPF19; 1.5-6-fold in phase 2 and/or phase $3 ; p$-value $=0.0003$ 0.048), and the same observation was made for proteolytic-related proteins in phase 3 (SERPINA1 and PSMC3; 4-5-fold; $p$-value $=0.02-0.046$ ). The other SC proteins downregulated upon fasting vs. the fed state are known to be involved either in lipid metabolism (APOA1, 
APOA4, CESD1, ANXA6, and SNCG; 2-5-fold in phase 2 and/or phase 3 ; $p$-value $=0.003-0.04$ ) or in other functions like cell structure or iron transport (see Table S3).

\section{Plasma hemostasis-related factors in response to prolonged fasting}

Among adipose secreted factors, we notably observed changes concerning PAl1 expression in response to prolonged fasting (see above), PAl1 being well-known to control hemostasis. To gain further insights into fasting-induced changes in hemostasis-related mechanisms, we have analysed the plasma proteome from fed and fasted rats. Compared to the fed state, we found that the levels of plasma D-dimers were significantly increased by fasting (1.8-fold in phase 3 ), while the levels of fibrinopeptide A (FPA) and of a C-terminal degradation product from the fibrinogen $\alpha$-chain (c-ter FIBA) were significantly decreased (4.8-7.6-fold) in both fasting stages (Figure 4; see also Figures S4-S5 and Tables S4).

\section{Validation of EPI omics results using Western-blot analyses}

Western blot results, which are presented in Figure S6, confirmed several of the above reported results obtained using proteomics. Fasting-induced decreased levels in EPI carbonic anhydrase 3 (CA3) and endoplasmic reticulum resident protein 29 (ERP29) were found here to be more pronounced in phase 3 than phase 2 compared to the fed state (1.6-3.7 fold). The decrease reported above for protein levels of SC apolipoprotein A-IV (ApoA-IV) and endoplamsin (HSP90B1 or GRP94) was found to be also valid in EPI, where the drop was especially marked in phase 3 relative to the fed state (3.2-12-fold). However, significance was not reached for EPI ApoA-IV due to inter-individual variations. In EPI, the levels of the ApoAIV transcript were found to be elevated in phase 3 (see Table S1), which suggests post- 
transcriptional regulations. Finally, complementary to the decreased levels of ATP5B and ATP5H subunits of ATP synthase in EPI of fasted rats, no change was observed here regarding the levels of the ATP5A1 subunit. 


\section{Discussion}

Different metabolic phases have been described as the fast duration is extended, with lipid fuels (phase 2) and body proteins (phase 3 ) being successively used as the main source of energy. The prolonged fasting paradigm thus offers the opportunity to examine whether the adipose tissue responses differ according to fuel partitioning and/or the level of body reserve depletion. In the current paper, omics analyses highlighted positive correlations when comparing changes in protein versus transcript levels of the 35 entries that were common in both datasets. However, relationships were weak when considering changes in phase $2 \mathrm{vs.} \mathrm{the}$ fed state ( $r=0.51$ falling to $r=0.2$ when removing two exceptional values), changes in phase 3 vs. the fed state $(r=0.32)$, and changes in phase 3 vs. phase 2 of fasting $(r=0.04)$. In prolonged fasted rats compared to fed animals, differentially expressed transcripts and proteins highlight the same regulated processes/pathways (Figure 2). It was notably interesting to observe that both differentially expressed transcripts and proteins that are involved in carbohydrate metabolism and oxidative phosphorylation (OXPHOS) were globally downregulated in prolonged fasted rats compared to fed animals. Concerning the response to oxidative stress, transcripts were globally up-regulated whereas proteins were globally down-regulated. Due the relatively low number of differentially expressed proteins, the comparison of changes in transcripts and proteins involved in protein and lipid metabolisms is less obvious. As a consequence, we discuss separately hereafter these complementary data.

\section{Epididymal adipose tissue lipid metabolism during metabolic phases of prolonged fasting}

Fasting induced a very pronounced change of the omics profiles of adipose tissues. As revealed by functional annotation analysis of differential transcripts, lipid metabolism is obviously one of the main significantly altered processes in both phases of fasting. Most of lipogenic genes 
were downregulated by prolonged fasting vs. the fed state, often in more marked manner in phase 3 than phase 2, and a high number of lipolytic-related genes were found to be differentially expressed in fasted rats according to fuel partitioning. We have previously shown that adipose triglyceride lipase (ATGL) and hormone-sensitive lipase (HSL) are not coordinately regulated in response to changes in fuel partitioning during food deprivation (Bertile and Raclot, 2011). Data in the current study nicely accord with these previous results, and bring new information suggesting that monoglyceride lipase (MGL) is regulated as HSL is at the mRNA level. Among other lipid metabolizing enzymes, the increased mRNA levels of abhd6 in phase 3 and of abhd12 in phase 2 could be partly explained by the fact that they function not only as monoglyceride lipases, but also as lysophospholipases (Thomas et al., 2014). The action of lipases results in the generation of glycerol and fatty acids, which are released into the circulation. The decrease in mRNA levels of both the transporter of glycerol (AQP7) and of the transporters of fatty acids (FABPs), as well as the drop in NEFA levels during phase 3 of fasting vs. the fed state likely reflect low lipolytic rates at this stage.

A number of lipid droplet-associated proteins are known to modulate the rates of lipolysis (Duncan et al., 2007; Lafontan and Langin, 2009). The decrease in perilipin 1 (PLIN1) mRNA levels in phase 3 of fasting is in perfect line with previous results (Bertile and Raclot, 2011), and may indicate the importance of its transcriptional regulation in controlling lipid availability in late fasting. Perilipin2 (PLIN2), also known as adipose differentiation-related protein (ADRP) or adipophilin, has recently been reported to enhance lipolysis in adipocytes (Takahashi et al., 2016). Its increased mRNA levels in phase 3 of fasting could thus contribute, as do ATGL (see above), to maintain basal rates of lipolysis for sustaining body needs in lipid substrates in order to spare glucose and ketone bodies for other tissue requirements at this stage. ATGL activity is also known to be dose-dependently suppressed by G0/G1 switch gene 2 (GOS2) (Schweiger 
et al., 2012; X. Yang et al., 2010). Accordingly, we show here that the decrease in GOS2 expression, which has been reported at the protein level during short-term fasting (Zhang et al., 2014), is also valid at the mRNA level both in phase 2 and phase 3 of fasting. Finally, changes in other lipid droplet-associated proteins, namely cell death activator CIDE-3 (CIDEC or FSP27), caveolin-1 and seipin are also expected to reflect mechanisms whereby lipolysis is finely tuned during prolonged fasting.

Regulation pathways of lipolysis in adipocytes include the cAMP, cGMP, and insulin signalling pathways, along with the metabolism of arachidonic acid (Fruhbeck et al., 2014; Nielsen et al., 2014). Our data suggest that the transcriptional control of factors from all of these pathways is involved in the response to fuel partitioning during long term food deprivation. Interestingly, phospholipase C-related catalytically inactive protein (PRIP) knockout mice exhibit a lean phenotype (Yamawaki et al., 2017), which has recently led to conclude that PRIP may be involved in the regulation of lipolysis in white adipocytes. The more marked increase in PRIP mRNA levels in phase 3 versus phase 2 of fasting could thus contribute to limit lipolysis rates in late fasting to values close to basal levels. Finally, among biomarkers differentiallyexpressed only in late fasting, changes in galectin-12 (LGALS12) mRNA levels may constitute a regulatory step contributing to sustain triacylglycerol mobilization during phase 3 , as this protein regulates lipolysis, presumably by limiting cAMP levels to downregulate PKA signalling (R. Y. Yang et al., 2012).

\section{Epididymal adipose tissue secreted factors during metabolic phases of prolonged fasting}

Adipose tissue is now known to secrete hundreds of proteins, hence playing a role in a number of biological processes (Bluher and Mantzoros, 2015; Fasshauer and Bluher, 2015). In the current study, several secreted factors involved in lipid metabolism and insulin signalling were 
the most affected by prolonged fasting. The levels of adiponectin (AdipoQ), resistin, insulinlike growth factor 1 (IGF1), and leptin transcripts were especially reduced in phase 3 . In addition, the levels of apolipoprotein E (ApoE) were especially increased in late fasting. These data are in very good accordance with previous results obtained with another method in similar conditions (Bertile and Raclot, 2004a; Bertile et al., 2009). Here we deepen these data by showing that, relative to the fed state, rat plasma adiponectin remains essentially unchanged during fasting whatever its duration. Amongst other actions, adiponectin has been reported to promote fat storage and reduce lipolysis, although the exact mechanisms are not well understood (Tao et al., 2014). The reduced expression levels of ADIPOR2, the main adiponectin receptor in adipose tissue, could thus contribute to facilitation of lipolysis and reduction of lipogenesis during the two metabolic phases of fasting. The drop in phase 2 leptinemia was dramatically accentuated in phase 3 so that leptin became undetectable, which accords with previous results (Bertile and Raclot, 2004a). In addition, EPI leptin mRNA levels dropped in both phase 2 and phase 3 of fasting, independently of the duration of fasting. Earlier conclusions that falling leptin levels are likely involved in metabolic adaptation to phase 3 and in the alarm signal triggering refeeding behaviour in late fasting (Bertile and Raclot, 2004a, 2006; Bertile et al., 2009), are thus reinforced here. Because leptin is also known to exert insulin-sensitizing effects (Blüher, 2013), its gradual reduced levels could favour insulin resistance as the duration of fasting increases. Indeed, fasting triggers insulin resistance in most mammals, which allows glucose to be spared for the tissues that are unable to oxidize lipid fuels like the central nervous system. At the same time, insulinemia is decreased, which favours lipolysis (Houser et al., 2013; Martinez and Ortiz, 2017). Here we show that insulinemia remains at low levels in both phase 2 and phase 3 of prolonged fasting. Interestingly, glucocorticoids promote lipolysis (Djurhuus et al., 2002), and also induce insulin 
resistance (Purnell et al., 2009). The known rise in corticosteronemia in phase 2 and phase 3 rats compared to fed animals (Bertile and Raclot, 2004a), could then constitute another mechanism favouring lipid fuel mobilization during prolonged fasting. Finally, as it plays a role not only in inflammation, but also in insulin secretion by pancreatic $\beta$-cells (Revollo et al., 2007; Sun et al., 2013), increased visfatin mRNA levels in phase 2 and especially phase 3 of fasting compared to the fed state, could constitute a first regulatory step towards reduction of insulin secretion during prolonged food deprivation.

Epididymal adipose tissue and immunity and angiogenesis during metabolic phases of prolonged fasting

It has been shown that inflammation is triggered by adipose tissue expansion during obesity (Reilly and Saltiel, 2017). On the reverse, fasting is known to have beneficial effects on white adipose tissue inflammation (Chehimi and Eljaafari, 2017). Here we show that the scheme is more complicated since the mRNA levels of important activators of the immune system were either increased (TGFb2, IL6st, TNR1B) or decreased (Cxcr2, Dpp4, adipsin, Rbp4) in phase 3 or remained unchanged throughout the fast (TGFb1, TGFb3, CCL2, MIF, TNF $\alpha$, IL6). Consistently, the circulating levels of TNF $\alpha$ remained very stable across rat groups. Altogether, these data suggest that, despite selective changes for activators of the immune system reported above, the impact of fasting on white adipose tissue inflammation may not differ greatly according to changes in substrate availability and/or the level of adipose store depletion.

Most of angiogenesis-related factors were downregulated at the mRNA level in phase 3 vs. the fed state, which could be consistent with the fact that adipose tissue microcirculation is less crucial in late fasting as lipid fuels are less mobilized. An alternative explanation could be 
that a decrease in adipose blood flow lowers the availability of lipid-derived fuel in late fasting, thereby indirectly contributing to the rise in protein breakdown of tissues like, e.g., the skeletal muscles for sustaining body energy needs. Concerning the VEGF growth factor family, only VEGFB mRNA levels were decreased in phase 3 while those of VEGFA and VEGFC remained unchanged. Contrary to VEGFB, VEGFA and VEGFC are able to bind to the receptor VEGFR-2, which generates stronger signals than VEGFR-1 (Shibuya, 2011). This could help sustaining angiogenesis capacity in the microenvironment of adipose tissue in late fasting, which could be part of mechanism facilitating basal lipolysis and possibly anticipating refeeding to efficiently restore fat reserves.

\section{Epididymal adipose tissue and hemostasis during metabolic phases of prolonged fasting}

Plasminogen activator inhibitor 1 (PAI) is a multifunctional protein, which is notably able to inhibit plasminogen activators (Yasar Yildiz et al., 2014). Thus, the gradual increase of PAI1 mRNA levels during prolonged fasting compared to the fed state may be involved in the reduction of fibrinolysis and could represent a risk of thrombosis and fibrosis on the long term. From the peptide mass profiling performed on rat plasma, the sequence of the C-terminal degradation product (C-ter FIBA) we identified likely corresponds to the so-called $\alpha \mathrm{C}$ domain, which is released from fibrinogen upon fibrin formation, and which promotes clot stabilization (Collet et al., 2005). In line with the decreased coagulation observed previously in fasting men (Muliar et al., 1984), the reduced levels of plasma fibrinopeptide $A$ and fibrinogen $\alpha C$ domain we observed here could reflect reduced fibrin formation in phase 2 and phase 3 fasted rats. Contrasting data have previously reported an increase of plasmatic fibrinolysis during subtotal fasting in healthy subjects, with PAl1 levels remaining unchanged (Huber et al., 2005). D-dimer is recognized as a specific end-product derived from plasmin-induced degradation of cross- 
linked fibrin (Soomro et al., 2016). Its increased plasma levels in late fasting, in a context where fibrin formation is expected to be reduced (see above), might be involved in the prevention of any risk of intravascular coagulation and thrombus formation during prolonged food deprivation and it may also help favouring lipid-derived fuel to be delivered to organs.

Among other biomarkers differentially-expressed only in late fasting, changes in Ass1 levels could help white adipose cells to better cope with the lack of dietary Arg and GIn in late fasting (Long et al., 2017). The levels of EPI Trim63 (E3 ubiquitin-protein ligase also known as Murf1) mRNA rose independently of the duration of fasting, but more markedly in phase 3 than in phase 2. As already hypothesized for the E3 ubiquitin ligase COP1 in white and brown adipocytes during short term fasting (Nakai et al., 2008; Qi et al., 2006), and provided that enhanced Trim63 transcript abundance is translated into higher abundance/activity at the protein level, it could be that TRIM63-derived ubiquitination of a variety of adipose proteins is required to promote energy savings and regulate lipid metabolism to maintain energy homeostasis, especially in late fasting. Finally, annexin 2 (ANXA2) is known to mediate fatty acid uptake into adipocytes (Salameh et al., 2016), and the exacerbated decrease in its protein level during phase 3 relative to phase 2 may thus favour lipid mobilisation over reesterification process in a more marked manner in late fasting.

\section{Regional differences in the response of EPI and SC adipose tissues to prolonged food deprivation}

Both EPI and SC adipose tissues were essentially characterized by diminished protein levels, with data suggesting lipid trafficking might be more affected in SC, while fasting-induced oxidative stress would be more marked and energy production more reduced in internal fat pads. Consistently, the metabolic activity of fat pads has been reported to depend on their 
anatomical location (Kwok et al., 2016). Visceral adipose tissue has been reported to be preferentially mobilized over subcutaneous adipose tissue during food restriction, total fasting (Bertile and Raclot, 2004b; Sugden et al., 1994; Tang et al., 2017), and even during modest weight loss (Chaston and Dixon, 2008). In the current study, very few protein levels differed between EPI and SC in fed animals, but responses to fasting highlighted several differences. For example, expression levels of two proteins involved in the transport of lipids (ApoA1 and ApoA4) and of the CESD1 lipase were lower in SC during fasting, whereas one protein involved in lipid transport was more abundant in EPI during fasting (ApoA1). Such differences between SC and EPI nicely accord with the preferential mobilization of internal fat pads during food deprivation. 


\section{Conclusion}

The current data highlight a regulatory transcriptional program whereby lipolytic-related factors including lipases, lipid droplet-associated and regulating pathway factors appeared selectively regulated during metabolic phases of fasting. The gene expression levels of adipose secreted factors and their receptors, along with the plasma levels of few of these factors could reflect the repression of insulin signalling during prolonged fasting. Leptin and adiponectin were highlighted as important factors that could, respectively, favour food behaviour in late fasting and control lipid metabolism during both phase 2 and phase 3. Future work deciphering post-translational modifications of lipid metabolism-related factors should prove useful in complementing the regulation pattern of fasting-responsive pathways. Additionally, determining how fasting-induced regulations in white adipose tissues are reversed by refeeding, notably after phase 2 versus phase 3 of fasting, would help better manage body reserve replenishment in pathophysiological situations. Finally, deciphering the molecular responses of oxidative tissues would be helpful to better understand fuel utilization in severely undernourished organisms. 


\section{Materials and methods}

\section{Ethical statement}

The study was conducted in strict accordance with the recommendations stated in the Public Health Service policy on Human Care and Use of Laboratory Animals. All experiments were performed in conformity with the rules of the European Committee Council Directive of November 24, 1986 (86/609/EEC) and the French Department of Agriculture (license no. 67226 to T.R.).

\section{Animals and study design}

Twenty-four male Sprague Dawley rats (Janvier CERJ, Le Genest-St-Isle, France) were housed individually in a temperature-controlled room $\left(25 \pm 1^{\circ} \mathrm{C}\right)$, with constant photoperiod (12:12, light/dark). They were given free access to water and to a standard diet (UAR A04, Villemoisson, France, $50 \%$ carbohydrate, $5 \%$ fat, and $24 \%$ protein, in mass percentage). This diet was recommended for rearing laboratory rodents and met National Research Council requirements, as certified by the manufacturer. The rats were allowed to acclimatize for several days before entering the fasting protocol. They were weighed daily during the whole duration of the experiment. Water was supplied ad libitum throughout the experiment for all animals.

When reaching $\sim 280 \mathrm{~g}$, the rats (9-weeks old) were randomly divided into three groups ( $\mathrm{N}=8 /$ group). Eight rats were immediately sacrificed as control-fed animals by rapid decapitation, without prior anaesthesia (procedure necessary because anaesthesia could affect hormone, especially the stress hormone corticosterone, and lipid metabolism). These controls rats, which are referred to as fed animals thereafter, were in the postabsorptive state 
and had a full stomach at the time of sampling. The other rats were fasted until phase $2(\mathrm{~N}=8)$ and phase $3(\mathrm{~N}=8)$ of fasting. The rate of body mass loss $(\mathrm{dm} / \mathrm{m} . \mathrm{dt})$, which is known to reflect changes in the rate of protein utilization, was calculated from daily weighing. As phase 2 of prolonged fasting is characterized by protein sparing and phase 3 by an increase in body protein breakdown, mass loss data enabled us to identify the phase $2 /$ phase 3 transition as previously reported (Bertile et al., 2009; Bertile and Raclot, 2011; Cherel et al., 1991; Cherel and Le Maho, 1991). The metabolic status of rats was then validated further by plasma metabolite and hormone measurements ( $\mathrm{N}=8 /$ group). Although survival is challenged by protein wasting during phase 3 , this phase has been shown to be reversible provided that food availability is restored rapidly (Cherel and Le Maho, 1991). In the present study, phase 3 rats were killed $\sim 1$ day after the phase $2-$ phase 3 transition, i.e. in early phase 3 . All animals were killed by cervical dislocation between $10 \mathrm{AM}$ and $12 \mathrm{PM}$. Truncal blood was collected in ethylenediaminetetraacetic acid (EDTA) tubes immediately after sacrifice, and plasma was separated by centrifugation ( $1500 \mathrm{~g}$ ) for $15 \mathrm{~min}$. at $4^{\circ} \mathrm{C}$ and kept frozen at $-80^{\circ} \mathrm{C}$ until analysis. Epididymal (EPI) and inguinal subcutaneous (SC) white adipose tissues were quickly dissected from the freshly killed rats and weighed, and then pieces were snap frozen in liquid nitrogen and stored at $-80^{\circ} \mathrm{C}$ until analysis.

\section{Plasma metabolite and hormone assays}

The levels of plasma metabolites and hormones were measured in fed and fasted rats ( $N=8$ /group, except for $D$-dimer levels where $N=6 /$ group). The kits for enzymatic determination of plasma urea and glucose were purchased from Sigma Diagnostics (St. Louis, MO, USA). Plasma non-esterified fatty acids (NEFA) levels were measured using a kit from 
Wako Chemicals GmbH (Neuss, Germany), and the turbidimetric determination of cross-linked fibrin degradation products (D-dimer) using the D-Dimer PLUS kit from Siemens (Marburg, Germany). Commercial ELISA kits were used to measure plasma levels of leptin and adiponectin (Alpco Diagnostics; Salem, NH, USA), insulin (Merk Millipore, Molsheim, France), NPY (BioSupply, Shipley, UK) and TNF $\alpha$ (Sigma Diagnostics).

\section{Epididymal adipose tissue microarray analysis}

After homogenization of frozen EPI samples ( $\mathrm{N}=4$ /group) using a laboratory ball mill, total RNAs were prepared using the Trizol ${ }^{\circledR}$ Reagent, following the manufacturer's instructions (ThermoFisher Scientific). The quantity, quality, and purity of extracted RNA were assessed using a NanoDrop 1000 spectrophotometer (NanoDrop, Wilmington, DE, USA) at 260 and 280 $\mathrm{nm}$ and an Agilent 2100 bioanalyser (Agilent, Santa Clara, CA, USA). RNAs were considered pure for values of A260/280 ratio > 1.8 and of RNA Integrity Number (RIN) > 9 .

DNA microarray (Affymetrix GeneChip technology) analyses were carried out within the IGBMC (Institute of Genetics and Molecular and Cellular Biology, Illkirch, France) Microarray and Sequencing platform (http://www-microarrays.u-strasbg.fr/). Four separate hybridization sets were performed for the three experimental groups, using the GeneChip Rat Gene 1.0 ST array. All assays were performed as per instructions in Affymetrix GeneChip ${ }^{\circledR}$ Whole Transcript (WT) Sense Target Labeling Assay Manual (P/N 701880 Rev. 2) (http://www.affymetrix.com/support/technical/byproduct.affx?product=raexon-st). Microarrays were scanned using an Affymetrix GeneChip Scanner 3000 7G (Affymetrix UK Ltd., High Wycombe, UK). 
Quantification and initial analysis were done using the GeneChip Operating v1.4 (GCOS) and Expression Console v1.1 softwares (Affymetrix UK Ltd., High Wycombe, UK). Expression levels were calculated using the following parameters: sketch quantile for normalization, Median polish as in RMA for summarization. Subsequent analysis was performed on the extended set of probesets. Reference genes were eliminated.

The microarray data obtained in this publication have been deposited in NCBI's Gene Expression Omnibus and are accessible through GEO Series accession number GSE118978 (https://www.ncbi.nlm.nih.gov/geo/query/acc.cgi?acc=GSE118978).

\section{Epididymal and subcutaneous adipose tissue proteomics analysis}

Methods are detailed in Supplementary materials and methods. Proteomics analysis was performed as three different experiments to examine the effects of fasting in EPI (Experiment 1) and in SC (Experiment 2), and to compare protein profiles in EPI and SC control fed animals (Experiment 3).

After protein extraction, the samples were labelled using a CyDye DIGE Fluor Minimal Dye Labeling Kit (GE HealthCare, Uppsala, Sweden) and subjected to isoelectric focusing (IEF) and then sodium dodecyl sulfate (SDS)-PAGE electrophoresis. Gel images were acquired using an Ettan DIGE Imager (GE HealthCare) at $100 \mu \mathrm{m}$ resolution, and they were analysed using the PDQuest $^{T M}$ 2-D gel analysis software (v8.0.1; BioRad). For EPI (Experiment 1 ) and SC (Experiment 2), intensity values for phase $2(N=4)$ and phase $3(N=4)$ animals were normalized to intensities obtained for fed animals $(\mathrm{N}=4)$ which were assigned an arbitrary mean value of one. For the comparison of EPI and SC (Experiment 3), SC intensity values ( $N=$ 4) were normalized to intensities obtained in EPI $(N=4)$ which were assigned an arbitrary 
mean value of one. After statistical analysis (see below), differential protein spots were excised using an automated gel cutter (PROTEINEER sp, Bruker Daltonics, Bremen, Germany) and destained. In-gel reduction and alkylation of proteins were then performed using a Massprep Station (Waters, MicroMass, Manchester, UK) as previously reported (Plumel et al., 2014). Proteins were in-gel digested using trypsin (Promega, Madison, WI, USA). The resulting peptides were then analysed on a 1200 series nanoHPLC-Chip system (Agilent Technologies, Palo Alto, CA, USA) coupled to an $\mathrm{HCT}^{\mathrm{TM}}$ Plus ion trap (Bruker Daltonics, Bremen, Germany). Tandem mass spectrometry (MS/MS) data were analysed using the Mascot ${ }^{\mathrm{TM}}$ search engine (v2.5.1, Matrix Science, London, UK) installed on a local server. Spectra were searched against a target-decoy version of the Rattus norvegicus protein database downloaded from Swissprot (January 2016, 16118 target + decoy entries) to which common contaminants (e.g. trypsin and keratins) were automatically added using the MSDA software suite (Carapito et al., 2014). Stringent filtering criteria were applied using Proline software (v1.3; http://proline.profiproteomics.fr/) to obtain high confidence identifications (FDR $<1 \%$ ). Single-peptide-based protein identifications, as well as the identification of common contaminants such as keratin and trypsin, were not considered. The mass spectrometry proteomics data have been deposited to the ProteomeXchange Consortium via the PRIDE (Vizcaino et al., 2016) partner repository with the dataset identifier PXD010902.

Among the different proteins that were identified in a given spot, only the major (more abundant) ones were considered to be responsible for variations of spot intensities. The determination of major proteins was performed following a "peptide counting" strategy: the higher the number of peptides assigned to a given protein, the more abundant this protein. Here we took into account the fact that tryptic sites are followed or not by a Proline, the possible missed cleavages and the adequate size of peptides for their detection by mass 
spectrometry (i.e. peptides of 7 to 31 amino acids on the basis of our data). Then we compared the theoretical number of detectable tryptic peptides to the experimental number we identified. The theoretical number of detectable tryptic peptides was similar for major (54 \pm $5)$ and minor $(55 \pm 4)$ proteins in a given gel spot, and major proteins were identified with three times more peptides than minor ones ( $26 \pm 2$ vs. only $9 \pm 1$, respectively).

\section{Plasma MS/MS data analysis}

Methods are detailed in Supplementary materials and methods. Briefly, plasma samples ( $N=4 /$ group) were fractionated using a method based on solid phase extraction (SPE). Fractions were then spotted onto AnchorChip ${ }^{\mathrm{TM}}$ targets (Bruker Daltonics Gmbh, Bremen, Germany), and peptide mass profiling was achieved using a matrix-assisted laser desorptionionization (MALDI)-time-of-flight (TOF) mass spectrometer (ULTRAFLEX ${ }^{\mathrm{TM}}$, Bruker Daltonics Gmbh, Bremen, Germany). After alignment, integration of peak areas and comparison of peptide profiles, differential abundant peptides were identified after further fractionation of solid phase extraction (SPE) eluted fractions, using a capillary liquid chromatography (LC) system (Agilent 1100 series, Wilmington, DE, USA) equipped with a micro fraction collector (Agilent 1100 series micro fraction collector, Wilmington, DE, USA). The analysis of proteins from complex matrixes like the plasma is a challenging task. To obtain a complete set of information, including the detection and identification of differentially abundant proteins/peptides together with confirmation of their sequences, different methods were used. First LC-ESI-MS/MS of the peptides was performed on an Agilent 1100 Series capillary LC system coupled to a HCT $^{\mathrm{TM}}$ Plus ion trap (Bruker Daltonics Gmbh, Bremen, Germany). Second, direct infusion MS/MS experiments were performed in ESI mode using a $\mathrm{HCT}^{\mathrm{TM}}$ Plus ion trap (Bruker Daltonics Gmbh, Bremen, Germany). Third, analysis in nanoESI mode was 
done using a Q-Tof-2 ${ }^{\mathrm{TM}}$ (Waters, Milford, MA, USA) mass spectrometer. Fourth, MALDITOF/TOF experiments were performed on an ULTRAFLEX ${ }^{\text {TM }}$ mass spectrometer (Bruker Daltonics Gmbh, Bremen, Germany). Fifth, analysis by automated Edman degradation used a cLC microsequencer (Applied Biosystems, Foster City, Ca, USA).

\section{Bioinformatics analysis}

Hierarchical clustering was performed using the Cluster v3.0 software (de Hoon et al., 2004). Parameters were set as follows: median centering and normalization of genes for adjusting data and centroid linkage clustering for both genes and arrays. Dendrograms were generated and visualised using the Treeview v1.1.3 program.

Enrichment and functional annotation analysis of transcriptomics data was performed using the desktop version of DAVID (Ease v2.1) and an updated version of Gene Ontology (GO) databases (November 2016). Enriched GO terms were filtered by only considering those with an Ease score lower than 0.1, a Benjamini p-value lower than 0.05 and a fold enrichment higher than 2. Enriched GO terms were grouped together into broad functional categories, which were then considered as enriched broad functions.

\section{Statistical analysis}

Statistical analysis was performed using the R software environment v3.0.2 (R Development Core Team, 2008). All data are presented as means \pm SEM of the indicated number of biological replicates. The normal distribution of the measured variables was assessed using the Shapiro-Wilk test ( $p$-value $>0.01$ ), and homoscedasticity using the Bartlett test ( $p$-value > 0.01). Rat characteristics and plasma parameters were compared using ANOVA followed by 
post-hoc Tukey tests or by Welch Two Sample t-tests when applicable. For EPI (Experiment 1) and SC (Experiment 2), protein spot intensities between fed and fasted animals were compared using ANOVA followed by post-hoc Tukey tests ( $p$-value $<0.05$ ). Protein spot intensities in EPI and SC control animals (Experiment 3) were compared using paired sample Student t-tests ( $p$-value $<0.05$ ). For transcriptomics data, significance was considered for both ANOVA post-hoc Tukey tests $p$-values $<0.0001$. 


\section{Acknowledgment}

This work was supported by the Agence Nationale de la Recherche (Programme Proteonutr ANR-05-BLAN-0069) and the French Proteomic Infrastructure (ProFI; ANR-10-INSB-08-03). During the tenure of this study, TW was supported by the Centre National de la Recherche Scientifique (CNRS) and the Bruker Daltonics Company. Technical assistance from Chrystel Husser and Dr. JM Strub was essential to DIGE experiment and plasma profiling, respectively. We also thank Drs Christelle Thibault-Carpentier, Doulaye Dembele and Violaine Alunni for involvement in transcriptomics experiments, and Dr. Patrick Guterl for bioinformatics assistance in functional annotation analysis. 


\section{References}

Bertile, F., Criscuolo, F., Oudart, H., Le Maho, Y., and Raclot, T. (2003). Differences in the expression of lipolytic-related genes in rat white adipose tissues. Biochem. Biophys. Res. Commun., 307, 540-546.

Bertile, F., and Raclot, T. (2004a). Differences in mRNA expression of adipocyte-derived factors in response to fasting, refeeding and leptin. Biochim. Biophys. Acta, 1683, 101-109.

Bertile, F., and Raclot, T. (2004b). mRNA levels of SREBP-1c do not coincide with the changes in adipose lipogenic gene expression. Biochem. Biophys. Res. Commun., 325, 827-834.

Bertile, F., and Raclot, T. (2006). Adipose-derived factors during nutritional transitions. Curr. Nutr. Food Sci., 2, 127-139.

Bertile, F., and Raclot, T. (2008). Proteomics can help to gain insights into metabolic disorders according to body reserve availability. Curr. Med. Chem., 15, 2545-2558.

Bertile, F., Schaeffer, C., Le Maho, Y., Raclot, T., and Van Dorsselaer, A. (2009). A proteomic approach to identify differentially expressed plasma proteins between the fed and prolonged fasted states. Proteomics, 9, 148-158.

Bertile, F., and Raclot, T. (2011). ATGL and HSL are not coordinately regulated in response to fuel partitioning in fasted rats. J. Nutr. Biochem., 22, 372-379.

Bertile, F., Fouillen, L., Wasselin, T., Maes, P., Le Maho, Y., Van Dorsselaer, A., and Raclot, T. (2016). The Safety Limits Of An Extended Fast: Lessons from a Non-Model Organism. Sci. Rep., 6, 39008.

Bluher, M., and Mantzoros, C. S. (2015). From leptin to other adipokines in health and disease: facts and expectations at the beginning of the 21st century. Metabolism, 64, 131-145.

Blüher, M. (2013). Importance of adipokines in glucose homeostasis. Diabetes Manage., 3, 389-400.

Carapito, C., Burel, A., Guterl, P., Walter, A., Varrier, F., Bertile, F., and Van Dorsselaer, A. (2014). MSDA, a proteomics software suite for in-depth Mass Spectrometry Data Analysis using grid computing. Proteomics, 14, 1014-1019.

Chaston, T. B., and Dixon, J. B. (2008). Factors associated with percent change in visceral versus subcutaneous abdominal fat during weight loss: findings from a systematic review. Int. J. Obes. (Lond), 32, 619-628.

Chehimi, M., and Eljaafari, A. (2017). Beneficial effects of fasting on white adipose tissue inflammation and metabolic syndrome in obese subjects: Review. Endocrinol. Metab. Int. J, 4, 00105.

Cherel, Y., Attaix, D., Rosolowska-Huszcz, D., Belkhou, R., Robin, J. P., Arnal, M., and Le Maho, Y. (1991). Whole-body and tissue protein synthesis during brief and prolonged fasting in the rat. Clin. Sci. (Lond), 81, 611-619.

Cherel, Y., and Le Maho, Y. (1991). Refeeding after the late increase in nitrogen excretion during prolonged fasting in the rat. Physiol. Behav., 50, 345-349.

Collet, J. P., Moen, J. L., Veklich, Y. I., Gorkun, O. V., Lord, S. T., Montalescot, G., and Weisel, J. W. (2005). The alphaC domains of fibrinogen affect the structure of the fibrin clot, its physical properties, and its susceptibility to fibrinolysis. Blood, 106, 3824-3830.

de Hoon, M. J., Imoto, S., Nolan, J., and Miyano, S. (2004). Open source clustering software. Bioinformatics, 20, 1453-1454.

Ding, H., Zheng, S., Garcia-Ruiz, D., Hou, D., Wei, Z., Liao, Z., Li, L., Zhang, Y., Han, X., Zen, K., Zhang, C. Y., Li, J., and Jiang, X. (2016). Fasting induces a subcutaneous-to-visceral fat switch mediated by microRNA-149-3p and suppression of PRDM16. Nat. Commun., 7, 11533.

Djurhuus, C. B., Gravholt, C. H., Nielsen, S., Mengel, A., Christiansen, J. S., Schmitz, O. E., and Möller, N. (2002). Effects of cortisol on lipolysis and regional interstitial glycerol levels in humans. Am. J. Physiol., 283, E172-E177.

Duncan, R. E., Ahmadian, M., Jaworski, K., Sarkadi-Nagy, E., and Sul, H. S. (2007). Regulation of lipolysis in adipocytes. Annu. Rev. Nutr., 27, 79-101. 
Duszka, K., Bogner-Strauss, J. G., Hackl, H., Rieder, D., Neuhold, C., Prokesch, A., Trajanoski, Z., and Krogsdam, A. M. (2013). Nr4a1 Is Required for Fasting-Induced Down-Regulation of Ppar gamma 2 in White Adipose Tissue. Mol. Endocrinol., 27, 135-149.

Fasshauer, M., and Bluher, M. (2015). Adipokines in health and disease. Trends Pharmacol. Sci., 36, 461-470.

Faulconnier, Y., Chilliard, Y., Torbati, M. B. M., and Leroux, C. (2011). The transcriptomic profiles of adipose tissues are modified by feed deprivation in lactating goats. Comp. Biochem. Physiol. D-Genomics Proteomics, 6, 139-149.

Fruhbeck, G., Mendez-Gimenez, L., Fernandez-Formoso, J. A., Fernandez, S., and Rodriguez, A. (2014). Regulation of adipocyte lipolysis. Nutr. Res. Rev., 27, 63-93.

Groscolas, R., and Robin, J. P. (2001). Long-term fasting and re-feeding in penguins. Comp. Biochem. Physiol. A. Mol. Integr. Physiol., 128, 645-655.

Houser, D. S., Champagne, C. D., and Crocker, D. E. (2013). A non-traditional model of the metabolic syndrome: the adaptive significance of insulin resistance in fasting-adapted seals. Front. Endocrinol. (Lausanne), 4, 164.

Huber, R., Nauck, M., Basler, N., Haas, B., Mattern, M., Ludtke, R., and Peter, K. (2005). Effects of subtotal fasting on plasmatic coagulation, fibrinolytic status and platelet activation: a controlled pilot study in healthy subjects. Nut. Metab. Cardiovasc. Dis., 15, 212-218.

Ji, B., Ernest, B., Gooding, J. R., Das, S., Saxton, A. M., Simon, J., Dupont, J., Metayer-Coustard, S., Campagna, S. R., and Voy, B. H. (2012). Transcriptomic and metabolomic profiling of chicken adipose tissue in response to insulin neutralization and fasting. Bmc Genomics, 13, 441.

Kwok, K. H., Lam, K. S., and Xu, A. (2016). Heterogeneity of white adipose tissue: molecular basis and clinical implications. Exp. Mol. Med., 48, e215.

Lafontan, M., and Langin, D. (2009). Lipolysis and lipid mobilization in human adipose tissue. Prog. Lipid Res., 48, 275-297.

Li, R. Y., Zhang, Q. H., Liu, Z., Qiao, J., Zhao, S. X., Shao, L., Xiao, H. S., Chen, J. L., Chen, M. D., and Song, H. D. (2006). Effect of short-term and long-term fasting on transcriptional regulation of metabolic genes in rat tissues. Biochem. Biophys. Res. Commun., 344, 562-570.

Long, Y., Tsai, W. B., Wang, D., Hawke, D. H., Savaraj, N., Feun, L. G., Hung, M. C., Chen, H. H., and Kuo, M. T. (2017). Argininosuccinate synthetase 1 (ASS1) is a common metabolic marker of chemosensitivity for targeted arginine- and glutamine-starvation therapy. Cancer Lett., 388, 54-63.

Luo, L., and Liu, M. (2016). Adipose tissue in control of metabolism. J Endocrinol, 231(3), R77-R99.

Manzoni, C., Kia, D. A., Vandrovcova, J., Hardy, J., Wood, N. W., Lewis, P. A., and Ferrari, R. (2016). Genome, transcriptome and proteome: the rise of omics data and their integration in biomedical sciences. Brief Bioinform., 19, 286-302.

Martinez, B., and Ortiz, R. M. (2017). Thyroid Hormone Regulation and Insulin Resistance: Insights From Animals Naturally Adapted to Fasting. Physiology (Bethesda), 32, 141-151.

Martinez, B., Khudyakov, J., Rutherford, K., Crocker, D. E., Gemmell, N., and Ortiz, R. M. (2018). Adipose transcriptome analysis provides novel insights into molecular regulation of prolonged fasting in northern elephant seal pups. Physiol. Genomics, 50, 495-503.

Muliar, L. A., Mishchenko, V. P., Loban, G. A., Goncharenko, L. L., and Bobyrev, V. N. (1984). Effect of complete fasting on the coagulative and antioxidative properties of blood. Vopr. Pitan., 4, 2023.

Nakai, Y., Hashida, H., Kadota, K., Minami, M., Shimizu, K., Matsumoto, I., Kato, H., and Abe, K. (2008). Up-regulation of genes related to the ubiquitin-proteasome system in the brown adipose tissue of 24-h-fasted rats. Biosci. Biotechnol. Biochem., 72, 139-148.

Nielsen, T. S., Jessen, N., Jorgensen, J. O., Moller, N., and Lund, S. (2014). Dissecting adipose tissue lipolysis: molecular regulation and implications for metabolic disease. J. Mol. Endocrinol., 52, R199-222. 
Owen, O. E., Smalley, K. J., D'Alessio, D. A., Mozzoli, M. A., and Dawson, E. K. (1998). Protein, fat, and carbohydrate requirements during starvation: anaplerosis and cataplerosis]. Am. J. Clin. Nutr., 68, 12-34.

Palou, M., Sanchez, J., Priego, T., Rodriguez, A. M., Pico, C., and Palou, A. (2010). Regional differences in the expression of genes involved in lipid metabolism in adipose tissue in response to shortand medium-term fasting and refeeding. J. Nutr. Biochem., 21, 23-33.

Plumel, M. I., Stier, A., Thierse, D., van Dorsselaer, A., Criscuolo, F., and Bertile, F. (2014). Litter size manipulation in laboratory mice: an example of how proteomic analysis can uncover new mechanisms underlying the cost of reproduction. Front. Zool., 11, 41.

Purnell, J. Q., Kahn, S. E., Samuels, M. H., Brandon, D., Loriaux, D. L., and Brunzell, J. D. (2009). Enhanced cortisol production rates, free cortisol, and 11beta-HSD-1 expression correlate with visceral fat and insulin resistance in men: effect of weight loss. Am. J. Physiol. Endocrinol. Metab., 296, E351-357.

Qi, L., Heredia, J. E., Altarejos, J. Y., Screaton, R., Goebel, N., Niessen, S., Macleod, I. X., Liew, C. W., Kulkarni, R. N., Bain, J., Newgard, C., Nelson, M., Evans, R. M., Yates, J., and Montminy, M. (2006). TRB3 links the E3 ubiquitin ligase COP1 to lipid metabolism. Science, 312, 1763-1766.

R Development Core Team. (2008). R: A language and environment for statistical computing. $R$ Foundation for Statistical Computing, Vienna, Austria, ISBN 3-900051-07-0, URL http://www.R-project.orq.

Reilly, S. M., and Saltiel, A. R. (2017). Adapting to obesity with adipose tissue inflammation. Nat. Rev. Endocrinol., 13, 633-643.

Revollo, J. R., Korner, A., Mills, K. F., Satoh, A., Wang, T., Garten, A., Dasgupta, B., Sasaki, Y., Wolberger, C., Townsend, R. R., Milbrandt, J., Kiess, W., and Imai, S. (2007). Nampt/PBEF/Visfatin regulates insulin secretion in beta cells as a systemic NAD biosynthetic enzyme. Cell Metab., 6, 363-375.

Salameh, A., Daquinag, A. C., Staquicini, D. I., An, Z., Hajjar, K. A., Pasqualini, R., Arap, W., and Kolonin, M. G. (2016). Prohibitin/annexin 2 interaction regulates fatty acid transport in adipose tissue. JCl Insight, 1, e86351.

Schupp, M., Chen, F., Briggs, E. R., Rao, S., Pelzmann, H. J., Pessentheiner, A. R., Bogner-Strauss, J. G., Lazar, M. A., Baldwin, D., and Prokesch, A. (2013). Metabolite and transcriptome analysis during fasting suggest a role for the p53-Ddit4 axis in major metabolic tissues. Bmc Genomics, 14, 758.

Schweiger, M., Paar, M., Eder, C., Brandis, J., Moser, E., Gorkiewicz, G., Grond, S., Radner, F. P., Cerk, I., Cornaciu, I., Oberer, M., Kersten, S., Zechner, R., Zimmermann, R., and Lass, A. (2012). G0/G1 switch gene-2 regulates human adipocyte lipolysis by affecting activity and localization of adipose triglyceride lipase. J. Lipid Res., 53, 2307-2317.

Sethi, J. K., and Vidal-Puig, A. J. (2007). Thematic review series: adipocyte biology. Adipose tissue function and plasticity orchestrate nutritional adaptation. J. Lipid Res., 48, 1253-1262.

Shibuya, M. (2011). Vascular Endothelial Growth Factor (VEGF) and Its Receptor (VEGFR) Signaling in Angiogenesis: A Crucial Target for Anti- and Pro-Angiogenic Therapies. Genes Cancer, 2, 1097-1105.

Soomro, A. Y., Guerchicoff, A., Nichols, D. J., Suleman, J., and Dangas, G. D. (2016). The current role and future prospects of D-dimer biomarker. Eur. Heart J. Cardiovasc. Pharmacother., 2, 175184.

Sugden, M. C., Grimshaw, R. M., Lall, H., and Holness, M. J. (1994). Regional variations in metabolic responses of white adipose tissue to food restriction. Am. J. Physiol., 267, E892-899.

Sun, Z., Lei, H., and Zhang, Z. (2013). Pre-B cell colony enhancing factor (PBEF), a cytokine with multiple physiological functions. Cytokine Growth Factor Rev., 24, 433-442.

Takahashi, Y., Shinoda, A., Kamada, H., Shimizu, M., Inoue, J., and Sato, R. (2016). Perilipin2 plays a positive role in adipocytes during lipolysis by escaping proteasomal degradation. Sci. Rep., 6, 20975. 
Tang, H. N., Tang, C. Y., Man, X. F., Tan, S. W., Guo, Y., Tang, J., Zhou, C. L., and Zhou, H. D. (2017). Plasticity of adipose tissue in response to fasting and refeeding in male mice. Nutr. Metab. (Lond), 14, 3.

Tao, C., Sifuentes, A., and Holland, W. L. (2014). Regulation of glucose and lipid homeostasis by adiponectin: effects on hepatocytes, pancreatic beta cells and adipocytes. Best Pract. Res. Clin. Endocrinol. Metab., 28, 43-58.

Thomas, G., Brown, A. L., and Brown, J. M. (2014). In vivo metabolite profiling as a means to identify uncharacterized lipase function: Recent success stories within the alpha beta hydrolase domain, (ABHD) enzyme family. Biochim. Biophys. Acta Mol. Cell Biol. Lipids, 1841, 10971101.

Viscarra, J. A., and Ortiz, R. M. (2013). Cellular mechanisms regulating fuel metabolism in mammals: role of adipose tissue and lipids during prolonged food deprivation. Metabolism, 62, 889-897.

Vizcaino, J. A., Csordas, A., del-Toro, N., Dianes, J. A., Griss, J., Lavidas, I., Mayer, G., Perez-Riverol, Y., Reisinger, F., Ternent, T., Xu, Q. W., Wang, R., and Hermjakob, H. (2016). 2016 update of the PRIDE database and its related tools. Nucleic Acids Res 44, D447-456.

Wasselin, T., Zahn, S., Maho, Y. L., Dorsselaer, A. V., Raclot, T., and Bertile, F. (2014). Exacerbated oxidative stress in the fasting liver according to fuel partitioning. Proteomics, 14, 1905-1921.

Yamawaki, Y., Oue, K., Shirawachi, S., Asano, S., Harada, K., and Kanematsu, T. (2017). Phospholipase C-related catalytically inactive protein can regulate obesity, a state of peripheral inflammation. Jpn Dent. Sci. Rev., 53, 18-24.

Yang, L., Vaitheesvaran, B., Hartil, K., Robinson, A. J., Hoopmann, M. R., Eng, J. K., Kurland, I. J., and Bruce, J. E. (2011). The fasted/fed mouse metabolic acetylome: N6-acetylation differences suggest acetylation coordinates organ-specific fuel switching. J. Proteome Res., 10, 41344149.

Yang, R. Y., Havel, P. J., and Liu, F. T. (2012). Galectin-12: A protein associated with lipid droplets that regulates lipid metabolism and energy balance. Adipocyte, 1, 96-100.

Yang, X., Lu, X., Lombes, M., Rha, G. B., Chi, Y. I., Guerin, T. M., Smart, E. J., and Liu, J. (2010). The $\mathrm{G}(0) / \mathrm{G}(1)$ switch gene 2 regulates adipose lipolysis through association with adipose triglyceride lipase. Cell Metab., 11, 194-205.

Yasar Yildiz, S., Kuru, P., Toksoy Oner, E., and Agirbasli, M. (2014). Functional stability of plasminogen activator inhibitor-1. Sci. World J., 2014, 858293.

Zhang, X., Xie, X., Heckmann, B. L., Saarinen, A. M., Czyzyk, T. A., and Liu, J. (2014). Targeted disruption of G0/G1 switch gene 2 enhances adipose lipolysis, alters hepatic energy balance, and alleviates high-fat diet-induced liver steatosis. Diabetes, 63, 934-946. 
Table 1. Characteristics of fed and fasted rats

\begin{tabular}{|c|c|c|c|c|c|c|c|}
\hline State & Fed & Phase 2 & Phase 3 & \multicolumn{4}{|c|}{ p-values } \\
\hline Main fuels & $\operatorname{Mix}^{1}$ & Lipids & Proteins & ANOVA & P2 vs. fed & P3 vs. fed & $\mathrm{P} 3$ vs. $\mathrm{P} 2$ \\
\hline \multicolumn{8}{|c|}{ Rat profiles } \\
\hline Initial body mass (g) & $276.8 \pm 1.0$ & $276.6 \pm 1.9$ & $278.8 \pm 0.8$ & 0.44 & 0.99 & 0.54 & 0.48 \\
\hline Final body mass (g) & - & $213.7 \pm 3.5$ & $188.1 \pm 3.3$ & - & - & - & $1.2 \times 10^{-4}$ \\
\hline Body mass loss (\%) & - & $22.8 \pm 1.0$ & $32.5 \pm 1.1$ & - & - & - & $1.5 \times 10^{-5}$ \\
\hline $\mathrm{dm} / \mathrm{m} \cdot \mathrm{dt}\left(\mathrm{g} \cdot 100 \mathrm{~g}^{-1} \cdot \mathrm{d}^{-1}\right)$ & - & $8.4 \pm 0.7$ & $10.3 \pm 0.3$ & - & - & - & $2.8 \times 10^{-2}$ \\
\hline Uremia (mM) & $3.8 \pm 0.2$ & $5.0 \pm 0.4$ & $8.1 \pm 0.6$ & $3.0 \times 10^{-6}$ & 0.17 & $<1.0 \times 10^{-4}$ & $2.0 \times 10^{-4}$ \\
\hline Duration of fasting (d) & - & $3.0 \pm 0.0$ & $5.4 \pm 0.2$ & - & - & - & $3.7 \times 10^{-6}$ \\
\hline \multicolumn{8}{|c|}{ White adipose tissue } \\
\hline EPI mass (g) & $2.3 \pm 0.1$ & $1.3 \pm 0.1$ & $0.6 \pm 0.0$ & $5.6 \times 10^{-9}$ & $<1.0 \times 10^{-4}$ & $<1.0 \times 10^{-4}$ & $7.0 \times 10^{-4}$ \\
\hline EPI mass loss (\%) & - & $42.6 \pm 6.0$ & $73.9 \pm 2.1$ & - & - & - & $8.8 \times 10^{-4}$ \\
\hline
\end{tabular}

Values are the means \pm SEM ( $n=8$ animals per group). P-values are given for ANOVA, Tukey

and Welch Two Sample t-tests. P2 and P3: phase 2 and phase 3 of fasting; EPI: epididymal adipose tissue. ${ }^{1}$ Main fuels include carbohydrates, lipids and proteins. 


\section{Figure legends}

\section{Figure 1. Overview of EPI transcriptomic response to prolonged fasting in rats}

Differentially abundant epididymal adipose tissue (EPI) transcripts among rat groups (A: $\mathrm{P}<$ 0.0001 for ANOVA and P $<0.05$ for Tukey tests; see details in Table S1; N=4/group). Heatmaps produced by hierarchical clustering for differential transcripts ( $B$ : $P<0.0001$ for ANOVA and $P$ $<0.05$ for Tukey tests; red, black and green boxes: downregulated, intermediate and upregulated transcripts, respectively). Functional annotation analysis (see also Tables S1-S2) from differential transcripts (C-E; ANOVA p-value<0.0001) among rat groups revealed broad functions affected by fasting stages (P2: phase 2; P3: phase 3). ER, Endoplasmic reticulum; ECM, extracellular matrix.

Figure 2. Overview of regulated EPI transcripts and proteins in response to prolonged

\section{fasting in rats}

Differentially expressed epididymal adipose tissue (EPI) transcripts $(A: P<0.0001)$ and proteins $(B ; P<0.05)$ in response to fasting stages ( $P 2$ : phase 2; $P 3$ : phase 3; $N=4 /$ group). Grey areas cover fold changes in-between -1.5 and 1.5. Orange plain lines $(y=x+\log 2(1.6)$ and $y=x-$ $\log 2(1.6))$ represent the theoretical curves that would be obtained if regulations in P2 and P3 vs. the fed state were linear as a function of time (duration of fasting). The farther the transcripts or proteins from this line, the more specific their regulation to the metabolic state of phase 3. For each protein, corresponding gel spot $\mathrm{N}^{\circ}$ (see Figure S2) is given. Protein isoforms identified in several gel spots are underlined. See Table S1 and S3 for details about functional categorization of regulated transcripts and proteins. 


\section{Figure 3. Regulation of EPI lipid metabolism in response to prolonged fasting in rats}

The relative abundance of differentially abundant EPI transcripts among rat groups is shown with regard to lipolysis (A) and lipogenesis (B). Colour codes (see legend box) highlight changes in response to fasting stages (P2: phase 2; P3: phase 3; N=4/group). For differentially expressed proteins $(\mathrm{P}<0.05)$ in $\mathrm{P} 2$ or $\mathrm{P} 3$ versus the fed state, abundance changes are qualified as strong up- or down-regulations when the magnitude of variation is higher than 2-fold, while the others are qualified as mild up- or down-regulations. Levels similar to those in the fed state are coloured in white, while non-measured factors are coloured in grey. Detailed values can be found in Table S1. Few circulating metabolite and hormone levels (in yellow) were also assessed (means $\pm \mathrm{SEM} ; \mathrm{N}=8$ /group); Bars that do not share the same superscript letter are significantly different: $\mathrm{P}<0.05$ for ANOVA and Tukey tests). AA: Arachidonate; ANP: atrial natriuretic peptide; AMP: Adenosine 5'-monophosphate; cAMP: cyclic AMP; Cga/TSHb: glycoprotein hormones/thyroid stimulating hormone beta; cGMP: cyclic GMP (Guanosine 3',5'-monophosphate); DG: diacylglycerols; G: Glycerol; FA: fatty acids; MG: monoacylglycerols; NE/E: norepinephrine/epinephrine; NPY: Neuropeptide Y; PGE2: Prostaglandin E2 ; TG: triacylglycerols.

\section{Figure 4. Regulation of EPI secreted factors in response to prolonged fasting in rats}

The relative abundance of differentially abundant EPI transcripts among rat groups is shown with regard to adipose secreted proteins and their receptors. Colour codes (see legend box) highlight changes in response to fasting stages (P2: phase 2; P3: phase 3; N=4/group For differentially expressed proteins $(\mathrm{P}<0.05)$ in $\mathrm{P} 2$ or $\mathrm{P} 3$ versus the fed state, abundance changes are qualified as strong up- or down-regulations when the magnitude of variation is higher than 
2-fold, while the others are qualified as mild up- or down-regulations. Levels similar to those in the fed state are coloured in white, while non-measured factors are coloured in grey. Detailed values can be found in Table S1. Few circulating metabolite and hormone levels ( $\mathrm{N}=8$ /group), as well as hemostatic factors ( $\mathrm{N}=4-6$ /group), were also assessed (means $\pm \mathrm{SEM}$; in yellow); Bars that do not share the same superscript letter are significantly different: $\mathrm{P}<$ 0.05 for ANOVA and Tukey tests). FPA: fibrinopeptide A; C-ter FIBA: C-terminal degradation product from the fibrinogen $\alpha$-chain. 


\section{Additional information}

\section{Author Contributions}

Conceptualization of the study by TR and FB. Experiments and data analysis by MI, DA, TW,

TR and FB. Data interpretation and drafting the article by MI, TR and FB. Critical revision of the paper by AVD and YLM. All authors read and approved the final manuscript.

\section{Competing financial interests}

The authors declare no competing financial interests.

\section{Supplementary Materials}

Any additional information on data can be found in Tables S1-S4 and Figures S1-S6. 